Running Head: Phenomenal causality and personality dominance

Martarelli, C. S. (2018). On the link between phenomenal causality and personality dominance. Cognitive Processing. doi.org/10.1007/s10339-018-0874-5

\title{
On the link between phenomenal causality and personality dominance
}

\author{
Corinna S. Martarelli \\ Swiss Distance Learning University, Switzerland \\ University of Bern, Switzerland
}

Corinna Martarelli, Swiss Distance Learning University, 3900 Brig, Switzerland

E-mail: corinna.martarelli@fernuni.ch

\begin{abstract}
Acknowledgements
I thank Trix Cacchione for helpful discussions regarding this paper. Further I thank Julia Schneider for preparing the stimulus material, Fitim Arifaj, Barbara Germann, Joel Hartmann, Sina Jossen, Nathanael Jost for data collection, all participants for taking part in this study, and three anonymous reviewers for providing helpful comments on an earlier version of the manuscript.
\end{abstract}


Running Head: Phenomenal causality and personality dominance

\title{
On the link between phenomenal causality and personality dominance
}

\begin{abstract}
The present study investigated whether personality dominance is related to phenomenal causality. Recently, renewed attention has been given to dispositional theories, suggesting that the causal impression arises because people interpret collisions in terms of antagonistic action roles. Here, we examined the relation between personality dominance and the judgment of causality of ambiguous patterns of motion. The results revealed that dominance as a trait is associated with an increased tendency to infer causality in ambiguous displays. We found that participants with high dominance scores (measured by means of a questionnaire) gave significantly more causal judgements than participants with low dominance scores. Our findings highlight that people's understanding of causality is grounded in their experiences of action.
\end{abstract}

Keywords: phenomenal causality; collisions; dispositional theories; personality dominance; embodied approach. 
Running Head: Phenomenal causality and personality dominance

\section{On the link between phenomenal causality and personality dominance}

When viewing a launch event (a moving object A that hits a stationary object B, whereupon B starts moving), we have an immediate causal impression of A causing the motion of B. This specific experience, first described by Michotte (1946/1963), is referred to as phenomenal causality (also called causal impression, and interaction impression, see e.g., Hubbard, 2013). If several characteristics of the stimulus are met (e.g., the subsequent direction and velocity of object B must be within specific limits) a launch effect is perceived. The work of Michotte sparked the controversy regarding whether observers perceive causality or whether observers infer causality while viewing launch events. Michotte argued that we see causality just as we see forms. He thus rejected the claim that we infer causality from repeated observations of co-occurrence between events or from repeated interactions with objects.

Recently renewed interest has been given to the Aristotelian dispositional theory of causality in psychological research on everyday causal reasoning (for an overview see Waldmann, 2017). Dispositional theories model all kinds of causal relations as interaction sequences of agent and patient, thereby focusing on the dispositions that agent- and patient-objects have (e.g., the agent has the disposition to act and the patient has the disposition to experience the agent's action). The launch event is perceived as if it were an interaction, with object $\mathrm{A}$ in the role of the agent and object $\mathrm{B}$ in the role of the patient. An example of this dispositional account is force dynamics (Pinker, 2007; Talmy, 1988; White, 2009; Wolff, 2008). While both White (e.g., 2012, 2017) and Wolff and colleagues (e.g., Wolff \& Shepard, 2013, Wolff \& Thorstad, 2017) focus on physical forces as an intuitive model of causality, they take opposing stances on the role of experienced forces in causal perception and reasoning. In White's theory, the focus is on experiences of acting on objects (i.e., human as agent), whereas Wolff's theory focuses on experiences of being on the receiving end of forces (i.e., human as patient) as a source of causal understanding. Theories taking an embodied approach are a type of dispositional theories. An influential theory of embodiment is the conceptual metaphor theory of Lakoff and Johnson (1980, 1999). Lakoff and Johnson's theory focuses on goal-directed action and sense of agency as an intuitive model of causality. People experience themselves as active agents that bring about certain effects when interacting with inert objects in the world. It has been suggested that people do not only use the intuitive model of causality in the domain of mechanical acting on inert objects. Rather, they generalize the model to all kinds of causal relations in the psychological and social domains (Lakoff \& Johnson, 1980).

Muentener and Bonawitz (2017) discuss three underlying mechanisms of the experience of causality, namely: physical launch events, goal-directed action of the self and others, and covariation information. They 
Running Head: Phenomenal causality and personality dominance

suggest that these mechanisms are integrated in a domain-general causal reasoning mechanism throughout development. Thus, it is not solely the perception of forces (physical domain) that is at play in the experience of a causal launch, but also action in a much broader way (including agency and intentionality, i.e. the psychological and social domains). If the causative agent-patient-interactions model that relies on our experience truly determines the way we perceive causality, it is plausible that differences in experienced agency in social interactions influence causal perception and reasoning. Thus, we hypothesize that collisions will be perceived differently, based on the individual's experience of agency in social relations. In other words, we expect that dominant persons, who experience themselves as efficient causal agents in everyday life, would be more likely to interpret collisions in terms of causal relations. To measure personality dominance, we selected the Personality Research Form (PRF) dominance scale (Jackson, 1984), a well-validated self-report measure of dominance that contains 16 true/false items, such as "I try to control others rather than permit them to control me" and "I avoid positions of power over other people" (reverse-coded).

To examine the association between personality dominance and phenomenal causality we needed ambiguous stimuli. To achieve this ambiguity, we decided to manipulate the spatial overlap, as has previously been done, for example by Scholl and Nakayama (2002). They showed that if object A fully overlapped with object B at the point of contact, then the event would be perceived as a non-causal passing event. Scholl and Nakayama $(2002,2004)$ and Choi and Scholl (2004) manipulated the percentage of overlap between the two objects when object A stopped moving, and object B started moving (with object A overlapping object B). By using these kinds of events, they found that the occurrence of the launch effect could be influenced by the manipulation of the context of these events. When presented with a typical no-overlap launch event adjacent to and simultaneously with an ambiguous overlapped event, participants tended to perceive a launch effect in the ambiguous stimulus. The authors explained this finding as an effect of visual attention. Not only do the characteristics of the event influence the perception of causality, but also the allocation of attention influences the perception of causality.

In our study, we were expecting the percentage of spatial overlap to influence the causal judgment (as previously shown). However, based on the assumption that people's understanding of causality is grounded in their experiences of action, we additionally hypothesized that personality dominance would be associated with an increased tendency to infer causality in ambiguous displays. We reasoned that people with high levels of dominance would be more likely to perceive collision events as causal launch events rather than non-causal pass events. 
Running Head: Phenomenal causality and personality dominance

\section{Method}

\section{Participants}

Seventy-six participants took part in this study (43 women), ranging in age from 18 to 73 ( $M=27.5, S D$ $=12.1)$. The participants' occupations were classified as: student (57\%), commercial profession (5\%), graduate occupation (9\%), technicians (8\%), social work (4\%), other (e.g., retired) (17\%). Participants were naïve about the hypothesis of the experiment and gave written-informed consent. The study was approved by the ethics committee of the University of Bern and was conducted in accordance with the Declaration of Helsinki.

\section{Stimuli}

We used simple collision events in which a moving object A hits a stationary object B, whereupon B starts moving. We manipulated the percentage of overlap between the two objects, where overlap refers to the $\%$ of object B that is occluded at the point in time where object A stops and object B starts moving. The two objects were overlapped $50 \%, 55 \%, 60 \%, 65 \%, 70 \%, 75 \%, 80 \%, 85 \%$ and $90 \%$. Overlap ranged from 50 to $90 \%$ (centered on $70 \%$ ). We chose this range of overlap, because previous studies have illustrated that maximal ambiguity is achieved with $67 \%$ overlap (Choi \& Scholl, 2004). Each trial involved two colored disks, one red and the other green (counterbalanced). The color of the overlapping region was the color of object A. Motion was always left-to-right since the impression of causality is strongest in this orientation compared to other orientations (Michotte, 1946/1963). Object A started near the left border of the screen, while object B started centered in the screen. When object A occluded some portions of object B, object A stopped moving and object B started moving to the other side of the screen (right border of the screen). We used 2:1, 1:1, and 1:1.25 A:B speed ratios. We varied the speed ratio in order to make the task more interesting from the view point of participants. We chose speed ratios that have been shown to give rise to a robust impression of causality (Hubbard, 2013). The collision events lasted around $1.6 \mathrm{sec}$. (see Figure A for an illustration of the trials). The test phase included two blocks. In each block, 54 trials (six trials per overlap-condition, i.e., two per each speed ratio, one with object $\mathrm{A}=$ red and one with object $\mathrm{A}=$ green) were presented in random order. We thus obtained causality judgments for 108 trials for each participant. In the pretest, four full-overlap events ( $100 \%$ overlap, no causality perceived) and four no-overlap collision events ( $0 \%$ overlap, launch event, causality is perceived) were used (see Figure 1).

Insert Figure 1 about here 


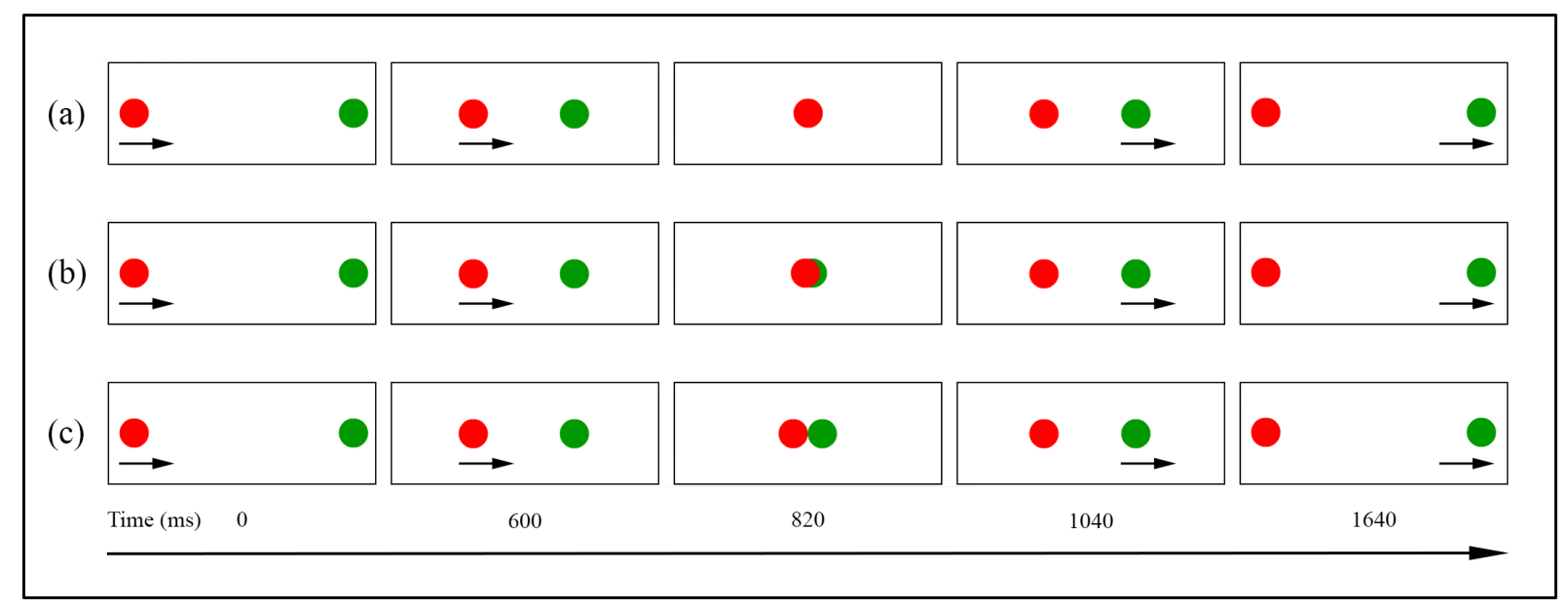

Figure 1. A graphical illustration of the trials. (a) a full overlap event (non-causal pass) (b) a $70 \%$ overlap event (ambiguous display) (c) a no-overlap event (causal launch).

\section{Questionnaire}

The 16 dominance scale items of the PRF (Jackson, 1984) were used to assess dominance. This scale is used to measure individuals' tendencies to e.g., make decisions. Participants judged 16 true/false statements such as "in an argument, I can usually win others over to my side". For each participant, we computed a dominance score (sum of dominant answers). The scale has shown high reliability (.96; Stumpf, Angleitner, Wieck, Jackson, \& Beloch-Till, 1985). As regards our sample, the Kuder-Richardson Formula 20 (1937) estimate of reliability was .76 for the 16 items, indicating acceptable internal consistency.

\section{Procedure}

All participants started with the test phase. Participants viewed the events and reported via key press whether they perceived the event as a causal launch (in which the first moving disk was seen to cause the motion of the second moving disk) or as a non-causal pass of one object by the other. The test phase included two blocks with 54 trials each, presented in random order. Before the test phase, participants completed a pretest phase with four full-overlap ( $100 \%$ overlap) and four no-overlap $(0 \%$ overlap) events presented in random order. In this phase participants were familiarized with the response buttons of the task and feedback was provided, as participants could only proceed to the next event if they had judged the no-overlap event as a causal launch and the full-overlap event as a non-causal pass. Since we were interested in the intuitive answer, we instructed participants to answer as fast as possible. After the test phase participants filled in the questionnaire. When filling in the questionnaire, participants can understand that we are interested in personality dominance; to avoid that this information changes how participants perceive the stimuli and interpret the task in the test phase, we chose a fixed order design (always the test phase before the questionnaire). 
Running Head: Phenomenal causality and personality dominance

\section{Results}

We used a median-split approach $(M d n=9)$ and considered participants scoring above the median as dominant $(\mathrm{n}=31)$, and participants scoring below the median as non-dominant $(\mathrm{n}=35)$. Even though we were aware of the problems related to median-split approaches, we decided to run a median-split analysis, mainly because of ease of communication. However, we conducted the same analysis with dominance as continuous variable in the model reported below and obtained similar results (no difference in rejecting the null hypothesis).

The dependent variable was the average proportion of trials on which launching was perceived, and this proportion was calculated for each overlap condition. Means are reported in Figure 2.

Insert Figure 2 about here

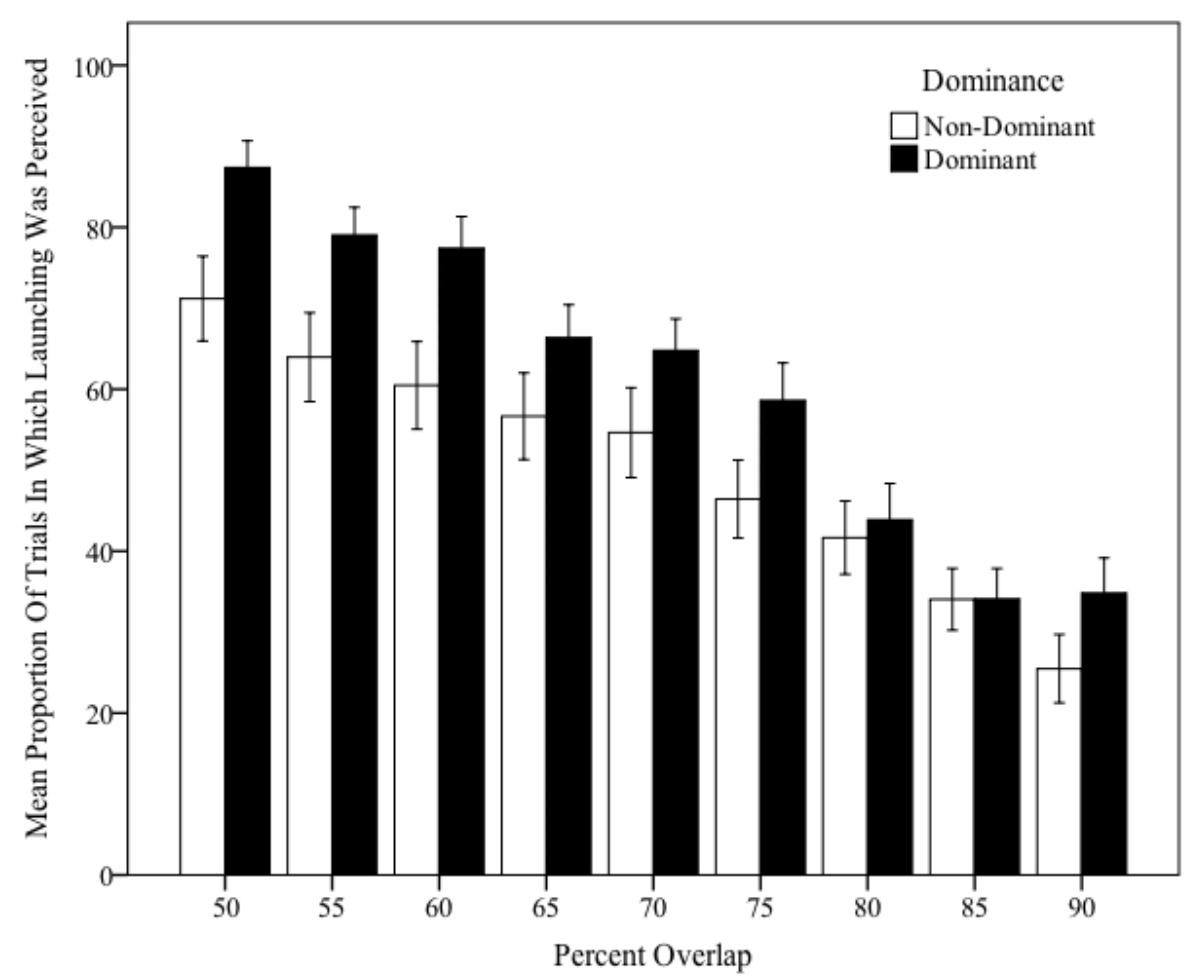

Figure 2. Proportion of trials in which launching was perceived by percent of overlap in the collision events and personality dominance of the participants. Error bars represent $+/-1$ standard error of the mean.

\section{Prediction of causality judgment by percent of overlap and personality dominance}

We computed a linear mixed-effects model with random intercepts for participants, and fixed effects for dominance (dominant vs. non-dominant group) and for percent of overlap in the collision events. Dominance was defined as level-2 predictor (person-specific) and percent of overlap as level-1 predictor (trial-specific). The degrees of freedom were obtained by a Satterthwaite approximation (Fai \& Cornelius, 1996) implemented in 
Running Head: Phenomenal causality and personality dominance

IBM SPSS Statistics, version 24. A full maximum likelihood method was used. Fixed effects are reported in Table 1. The results revealed a significant cross-level interaction, $F(1,528)=13.08, p<.001$. The estimated linear effect of the interaction was $-0.33(S E=0.09, p<.001)$. The significant interaction illustrated that the effect of dominance on causal judgment became weaker with increasing overlap; in other words, dominance played a role mainly in judging ambiguous stimuli and not in judging stimuli approaching the full overlap event (see $50,55,60$, and $65 \%$ of overlap in Figure 1).

The results also revealed a significant effect of dominance, $F(1,299.28)=15.66, p<.001$. Dominance was coded as 0 for non-dominant and as 1 for dominant. Given that the interaction term is in the model, this effect is a simple slope at baseline of percent of overlap ( $0 \%$ of overlap). To obtain interpretable simple slopes we centered percent of overlap on $60 \%, 70 \%$, and $80 \%$ (see Table 1). The estimated linear effect of dominance at $60 \%$ of overlap was $13.51(S E=5.56, p=.018)$, thus showing that the proportion of trials in which launching was perceived was $13.51 \%$ higher in dominant participants than in non-dominant participants when judging $60 \%$ overlapped collision events. This difference was significant. The estimated linear effect of dominance at $70 \%$ of overlap was $10.20(S E=5.49, p=.067)$, thus showing that the proportion of trials in which launching was perceived was $10.20 \%$ higher in dominant participants than in non-dominant participants when judging $70 \%$ overlapped collision events. This difference was marginally significant. The estimated linear effect of dominance at $80 \%$ of overlap was $6.89(S E=5.56, p=.219)$. This difference was not significant. When testing the simple slope at each percent of overlap, we found significant differences between dominant and non-dominant participants for the $65,60,55$, and $50 \%$ overlap ( $p$ s $<.036$, uncorrected $p$-values, see Appendix A).

The analysis also illustrated a significant effect of percentage of overlap, $F(1,528)=289.81, p<.001$. This effect is the simple slope of percentage of overlap at baseline of dominance $(0=$ non-dominant). The estimated linear effect of percentage of overlap was -1.07 ( $S E=0.06, p<.001)$, indicating a significant negative relationship between percentage of overlap and proportion of trials in which launching was perceived in the nondominant group (the higher the overlap the smaller the proportion of trials in which launching was perceived in the non-dominant group).

The most interesting result of this study is the significant interaction between percent of overlap and dominance. Thus, we correlated percent of overlap and percent of causal judgment for the dominant group and also for the non-dominant group. Pearson correlations confirmed that the more overlap an event included, the smaller the likelihood that it was judged as causal by participants (dominant group: $r(279)=-.631, p<.001$; non-dominant group: $r(315)=-.429, p<.001)$. Comparison of the two correlations yielded a significant 
Running Head: Phenomenal causality and personality dominance

difference $(z=3.44, p<.001)$. The negative correlation between percentage of overlap and causal judgments was significantly higher in the dominant group than in the non-dominant group, thus suggesting that the relation between percentage of overlap and causal judgments is stronger in the dominant group than in the non-dominant group.

Insert Table 1 about here

Table 1. Fixed effects for model predicting mean proportion of trials in which launching was perceived

\begin{tabular}{|c|c|c|c|c|c|c|c|c|c|c|c|c|}
\hline & \multicolumn{3}{|c|}{ No-centering } & \multicolumn{3}{|c|}{$\%$ overlap centered on 60} & \multicolumn{3}{|c|}{$\%$ overlap centered on 70} & \multicolumn{3}{|c|}{$\%$ overlap centered on 80} \\
\hline & Est. & SE & $p$ & Est. & SE & $p$ & Est. & SE & $p$ & Est. & SE & $p$ \\
\hline Intercept & 125.30 & 5.78 & $<.001$ & 61.19 & 3.81 & $<.001$ & 50.51 & 3.76 & $<.001$ & 39.82 & 3.81 & $<.001$ \\
\hline$\%$ Overlap & -1.07 & 0.06 & $<.001$ & -1.07 & 0.06 & $<.001$ & -1.07 & 0.06 & $<.001$ & -1.07 & 0.06 & $<.001$ \\
\hline Dominance & 33.38 & 8.44 & $<.001$ & 13.51 & 5.56 & .018 & 10.20 & 5.49 & .067 & 6.89 & 5.56 & .219 \\
\hline$\%$ Overlap x Dominance & -0.33 & 0.09 & $<.001$ & -0.33 & 0.09 & $<.001$ & -0.33 & 0.09 & $<.001$ & -0.33 & 0.09 & $<.001$ \\
\hline
\end{tabular}

overlap. Est. $=$ Estimate; $\mathrm{SE}=$ standard error.

\section{Discussion}

The results of the current study reveal that higher levels of personality dominance are associated with an increased tendency to experience causality in ambiguous displays. To the best of our knowledge, this is the first study reporting a relation between phenomenal causality and a personality variable. The effect of personality dominance that we found suggests that at least some individual difference variables can influence phenomenal causality. There is evidence for an impact of learning on phenomenal causality. For example, Peng and Knowles (2003) showed differences in phenomenal causality depending on the culture and education of participants (see also Bender, Beller, \& Medin, 2017). From the viewpoint of theories that concentrate on force mechanisms (e.g., White, 2006; Wolff, 2008) and on the experiential gestalt of causation (e.g. Lakoff \& Johnson, 1999), influence of experience and of individual differences on phenomenal causality can be predicted. Dispositional theories argue that causality is inferred from intuitive models, which are stored in long-term memory (White, 2012). Thus, they can be influenced by individual and cultural variables and can change with experience. One suggestion for why dispositional interpretations of causal sequences arise is that people use their own acting on objects as a model for causal relations (i.e. model them as causative agent-patient-interactions) and that this naïve model influences everyday causal inferences in different situations when interacting with inert objects and human beings (e.g., White, 2012). 
Running Head: Phenomenal causality and personality dominance

From a developmental point of view, it has been shown that the domain-general causal reasoning mechanism in adults derives from domain-specific constraints together with covariation information. As possible sources of domain-specific knowledge, physical launch events and goal-directed actions have been proposed (Muentener \& Bonawitz, 2017). Children do not represent collision events only in physical terms, but they are sensitive to goal-directed action and intentionality when observing the actions of others and when construing their own agency (e.g., Bélanger \& Desrocher, 2001, Schlottmann, Allen, Linderoth, \& Hesketh, 2002). Thus, it has been suggested that the source of causality understanding relies both on the physical and social domains. Our results indicate that the social domain might play a larger role in understanding causality than previously assumed. Moreover, our results suggest an overlap between physical and social domains. However, it has to be noted that we report a first result that needs to be replicated. The finding justifies the call for a more complete examination of the critical role that individual differences might play in causal reasoning.

The results reported in this study confirm that spatial overlap (characteristic of the stimulus) plays a role in phenomenal causality. The higher the overlap, the smaller the proportion of trials in which launching was perceived. It has to be noted, that we did not test overlaps of less than $50 \%$. We chose a range of overlap from $50 \%$ to $90 \%$ (centered on $70 \%$ ) because Choi and Scholl (2004) found maximal ambiguity at $67 \%$ overlap. Moreover, they illustrated that there is a tendency to see causality with high percentages of overlap. Their studies suggested that the perception of causality does not distribute symmetrically between $0 \%$ and $100 \%$ overlap. Future research is needed to test the effect of personality dominance at other percentages of overlap. It might be possible that effects of personality dominance would be strongest at maximal uncertainty rather than at smaller overlap.

Do high-dominant participants view collision events differently than do low-dominant participants or does the difference only pertain to the cognitive level (e.g. the judgement of causality)? Some authors have considered that we really see causality, whereas others argue that we infer causality, based on what we see, but also on what we know (Schlottmann, 2000). The relation between personality and causal impression that we found in our study suggests a role for experience and learning in causal understanding. It is possible that personality exerts direct, top-down modulations on what we see, however, another possibility is that individual differences appear after the perception at the level of cognition (judgement of causality). Although the current data do not allow us to conclude whether dominant vs. non-dominant participants perceive causality differently or infer causality differently, the current data do demonstrate that personality dominance might influence causal impressions. 
Running Head: Phenomenal causality and personality dominance

According to the literature, there are at least two ways to define dominance (e.g., Schultheiss, 2008); these are 1) dominance as a personality trait and 2) dominance as a person's hierarchical position (state). In the present study, dominance is understood as a personality trait. In future research, it would be interesting to investigate the role of power-state and felt power in the perceptual interpretation of causality. It would be possible to prime participants (high power priming vs. low power priming) or to manipulate power role as has been done in other research fields. For example, Bombari, Schmid Mast, and Bachmann (2017) asked participants to imagine being in either a high or a low power situation (i.e., participants had more or less power, respectively, over another person). Such an approach would help to better understand the underlying mechanism that we propose here, and, what is more, it would then allow a proper experimental manipulation with random assignment of participants to conditions. In our study, we used an individual differences perspective. Individual differences variables are problematic because participants are not assigned randomly to conditions. In other words, other variables might influence both personality dominance and causality judgement with ambiguous displays. Further research is needed to investigate other variables that are known to correlate with personality dominance, such as sense of agency (in terms of individual's awareness of what one can do). Visual inspection of Figure 2 reveals that the causality judgements of the non-dominant group declined almost linearly, while the judgements of the dominant group seemed to have a more jagged pattern of decline. It is conceivable that there are further sub-groups with varying thresholds for when causality is reported based on overlap in the group of dominant participants. This observation is consistent with the possibility that other variables influence both personality dominance and phenomenal causality. A further limitation of this study is that the dominance measure is based on a self-report measure. It might be useful to additionally assess dominance with more objective measures as in a social context. Moreover, it is important to further investigate whether these relationships between personality dominance and causal judgement impact real life and everyday causal judgements. Indeed, in our study, we limited the judgements to the domain of mechanical interactions. To summarize, this short report provides initial evidence that personality differences in dominance might influence how people perceive and/or reason in relation to causality when viewing collision events. These findings highlight that people's understanding of causality is grounded in their experiences of action. 
Running Head: Phenomenal causality and personality dominance

\section{References}

Bélanger, N., \& Desrochers, S. (2001). Can 6-month-old infants process causality in different types of causal events? British Journal of Developmental Psychology, 19, 11-21.

Bender, A., Beller, S., \& Medin, D. L. (2017). Causal cognition and culture. In M. R. Waldmann (Ed.), The Oxford Handbook of Causal Reasoning. Oxford University Press. Kindle-Version.

Bombari, D., Schmid Mast, M., \& Bachmann, M. (2017). Felt power explains the link between position power and experienced emotions. Emotion, 17(1), 55-66.

Choi, H., \& Scholl, B. J. (2004). Effects of grouping and attention on the perception of causality. Perception \& Psychophysics, 66, 926-942.

Fai, A. H. T., \& Cornelius, P. L. (1996). Approximate F-tests of multiple degree of freedom hypotheses in generalized least squares analyses of unbalanced split-plot experiments. The Journal of Statistical Computation and Simulation, 54(4), 363-378.

Hubbard, T. L. (2013). Phenomenal causality I: Variety and variables. Axiomathes, 23, 1-42.

Jackson, D.N. (1984). Personality research form manual (3rd ed.), Research Pyschologists Press, Port Huron, MI.

Kuder, G. F., \& Richardson, M. W. (1937). The theory of the estimation of test reliability. Psychometrika, 2, $151-160$.

Lakoff, G., \& Johnson, M. (1980). Metaphors we live by. Chicago: Chicago University Press.

Lakoff, G., \& Johnson, M. (1999). Philosophy in the flesh. New York: Basic Books.

Michotte, A. (1963). The perception of causality. (T. Miles \& E. Miles, Trans.). New York: Basic Books (original work published 1946)

Muentener, P. \& Bonawitz, E. (2017). Visual impressions of causality. In M. R. Waldmann (Ed.), The Oxford Handbook of Causal Reasoning. Oxford University Press. Kindle-Version.

Peng, K., \& Knowles, E. D. (2003). Culture, education, and the attribution of physical causality. Personality and Social Psychology Bulletin, 29, 1272-1284.

Pinker, S. (2007). The stuff of thought: Language as a window into human nature. New York: Viking.

Schlottmann, A. (2000). Is perception of causality modular? Trends in Cognitive Sciences, 4, 441-442.

Schlottmann, A., Allen, D., Linderoth, C., \& Hesketh, S. (2002). Perceptual Causality in Children. Child Development, 73(6), 1656-1677.

Scholl, B. J., \& Nakayama, K. (2002). Causal capture: contextual effects on the perception of collision events. 
Running Head: Phenomenal causality and personality dominance

Psychological Science, 13, 493-498.

Scholl, B. J., \& Nakayama, K. (2004). Illusory causal crescents: Misperceived spatial relations due to perceived causality. Perception, 33, 455-469.

Schultheiss, O. C. (2008). Implicit motives. In O. P. John, R. W. Robins \& L. A. Pervin (Eds.), Handbook of Personality: Theory and Research (3 ed., pp. 603-633). New York: Guilford.

Stumpf, H., Angleitner, A., Wieck, T., Jackson, D. N., \& Beloch-Till, H. (1985). Deutsche Personality Research Form (PRF). Handanweisung. Göttingen: Hogrefe.

Talmy, L. (1988). Force dynamics in language and cognition. Cognitive Science, 12, 49-100.

Waldmann, M. R. (2017). Causal reasoning: An introduction. In M. R. Waldmann (Ed.), The Oxford Handbook of Causal Reasoning. Oxford University Press. Kindle-Version.

White, P. A. (2006). The causal asymmetry. Psychological Review, 113, 132-147.

White, P. A. (2009). Property transmission: an explanatory account of the role of similarity information in causal inference. Psychological Bulletin, 135, 774-793.

White, P. A. (2012). Visual impressions of causality: effects of manipulating the direction of the target object's motion in a collision event. Visual Cognition, 20, 121- 142.

White, P. (2017). Visual impressions of causality. In M. R. Waldmann (Ed.), The Oxford Handbook of Causal Reasoning. Oxford University Press. Kindle-Version.

Wolff, P. (2008). Dynamics and the perception of causal events. In T. Shipley \& J. Zacks (Eds.), Understanding events: how humans see, represent, and act on events (pp. 555-587). New York: Oxford University Press.

Wolff, P., \& Shepard, J. (2013). Causation, touch, and the perception of force. In B. H. Ross (Ed.), The psychology of learning and motivation (pp. 167-202): Academic Press, Elsevier Inc.

Wolff, P., \& Thorstad, R. (2017). Force Dynamics. In M. R. Waldmann (Ed.), The Oxford Handbook of Causal Reasoning. Oxford University Press. Kindle-Version. 
Running Head: Phenomenal causality and personality dominance

Appendix A. Simple slope analysis at each percent of overlap that was assessed. Estimates (estimated linear effect of dominance) and uncorrected $p$-values are reported in the following table.

\begin{tabular}{lll}
\hline$\%$ of overlap centered on & Estimate of dominance & $p$-value \\
\hline 50 & 16.83 & .005 \\
55 & 15.17 & .009 \\
60 & 13.51 & .018 \\
65 & 11.86 & .035 \\
70 & 10.20 & .067 \\
75 & 8.55 & .125 \\
80 & 6.89 & .219 \\
85 & 5.24 & .357 \\
90 & 3.58 & .538 \\
\hline
\end{tabular}

